\title{
Spherical planetary nebulae
}

\author{
N. Soker \\ Department of Physics, University of Haifa at Oranim, Tivon 36006, Israel \\ Received 10 December 2001 / Accepted 19 February 2002

\begin{abstract}
By examining their mass loss history and their distribution in the galaxy, I argue that spherical planetary nebulae (PNe) form a special group among all planetary nebulae. The smooth surface brightness of most spherical PNe suggests that their progenitors did not go through a final intensive wind (FIW, also termed superwind) phase. While $\sim 70 \%$ of the PNe of all other PNe groups are closer to the galactic center than the sun is, only $\sim 30 \%$ of spherical PNe are; $\sim 70 \%$ of them are farther away from the galactic center. These, plus the well-known high scale height above the galactic plane of spherical PNe, suggest that the progenitors of spherical PNe are low mass stars having low metallicity. Although many stars have these properties, only $\sim 10 \%$ of all PNe are spherical. By comparing the galactic distribution of spherical PNe to the metallicity evolution in the galaxy, I find that the critical metallicity above which no spherical PNe are formed is $[\mathrm{Fe} / \mathrm{H}] \sim-0.4$. I explain this as well as other properties of spherical PNe in the context of the companion model for shaping PNe, arguing that spherical PNe are formed from stars that have no close companion, stellar or substellar, orbiting them. I discuss the connection of the proposed scenario to the recent finding of extrasolar planets and to the presence of blue horizontal branch stars in globular clusters.
\end{abstract}

Key words. planetary nebulae: general - stars: AGB and post-AGB - stars: mass loss - stars: planetary systems - stars: rotation

\section{Introduction}

Most planetary nebulae (PNe) have a large-scale axisymmetrical rather than spherical structure. Axisymmetrical PNe can be classified into two main groups; bipolar PNe, which are defined as PNe whose main structure contains two lobes with an equatorial waist between them, and elliptical PNe, which have a general elliptical shape, but no lobes, or only small ones. In the present Paper I refer to PNe having a large and dense concentration of mass in their equatorial plane, e.g., a ring, but without lobes, as extreme elliptical PNe. Whereas bipolar and elliptical $\mathrm{PNe}$ were the focus of many studies in recent years, basic questions regarding spherical $\mathrm{PNe}$ were not discussed much in the literature. The only point mentioned in some papers is a large scale height above (or below) the galactic plane of spherical $\mathrm{PNe}$ distribution, which implies that they are formed from low mass stars (e.g., Manchado et al. 2000). The question most often addressed is: what is the mechanism for forming non-spherical, i.e., bipolar or elliptical, structures? In the present Paper I reverse the emphasis by asking: what is required to form a spherical PN? Presenting the question this way allows me to consider a wider scope, e.g., the formation of blue horizontal branch (HB) stars. The two type of objects, I suggest, are connected in that both spherical PNe and blue HB stars are

\footnotetext{
* e-mail: soker@physics.technion.ac.il
}

formed from low mass stars, but blue HB stars will not form PNe. Therefore, the process behind the formation of blue HB stars will prevent a star from forming a PN.

Manchado et al. (2000) argue that $\sim 25 \%$ of the PNe in their sample are round. In the present Paper I use the term spherical PNe, assuming that the PNe I classified as spherical are indeed spherical and not pole-on elliptical or bipolar PNe. I use my earlier classification of spherical PNe (Soker 1997, hereafter S97), where more stringent criteria were used, and where only $18 \mathrm{PNe}$ are classified as spherical (Table 2 of S97). These amount to $4 \%$ of the 458 PNe classified there, but the estimate is that $\sim 10 \%$ of all PNe are spherical. Manchado et al. (1996) classify a $\mathrm{PN}$ as circular if its main large scale outer nebula is circular, sometimes not referring to the inner structure. I consider the inner structure as well, hence many PNe classified as circular by Manchado et al. (1996), are classified as elliptical in S97. A few examples are given below. Manchado et al. (1996) classify LSA 1 (PNG 029.8-07.8), K3-73 (PNG 084.0+09.5), and A 33 (PNG 238.0+34.8) as round $\mathrm{PNe}$, but I can clearly see two arcs within each of these PNe, which suggest that they are elliptical PNe. On the other hand, K1-14 (PNG 045.6+24.3) and A71 (PNG 084.9+04.4), which are classified as round PNe by Manchado et al. (1996), but not by S97, may have been spherical PNe before distortion. Another interesting case is A30 (PNG 208.5+33.2), which was classified as round 
by Manchado et al., but not by S97. The reason for the S97 classification of A30 as an elliptical PN is the knots close to its central star, that show an axisymmetrical structure. But beside these four knots the nebula shows all the properties of a spherical PN.

In Sect. 2 some aspects of the distribution of these spherical PNe in the galaxy and their mass loss history are studied. In Sect. 3 these properties are related to the metallicity of their progenitors. The strong correlation between PNe morphology, including spherical PNe, and metallicity holds for PNe in the Large Magellanic Cloud (LMC) as well (Stanghellini et al. 2000). In Sect. 3 I discuss also the formation of spherical $\mathrm{PNe}$ in relation to blue HB stars and to the more than 70 known extrasolar planets, arguing that the findings are compatible with the planet-induced axisymmetrical mass loss model. In this model low departure from spherical mass loss of some stars on the upper AGB are attributed to planets (Soker 2001a and references therein). A summary of the main results, and some speculations and predictions, are given in Sect. 4.

\section{Properties of spherical planetary nebulae}

\subsection{The galactic distribution}

It is well known that spherical PNe have a larger average galactic latitude $b$ than that of the other PNe (e.g., Manchado et al. 2000). I now show that the class of spherical PNe, as classified by S97, has also a larger galactic longitude than the other PNe. The $458 \mathrm{PNe}$ (of which 452 with good data are used here) that were classified by S97 were all the PNe available (in the literature) at that time that had good enough images to be classified according to their morphology. I use this list of PNe for the following reasons. (1) I deal here with morphologies of PNe, and this is the only large list of PNe where all PNe have wellresolved images. (2) I examine the hypothesis that planets may play a role. The classification of S97 takes the role of stellar companions, as well as planets, into consideration (see below). For example, considering a late interaction of a companion with the AGB progenitor, a $\mathrm{PN}$ that has a large spherical halo but an inner elliptical region is classified as an elliptical, rather than round PN. (3) Due to large uncertainties in the distances to $\mathrm{PNe}$, and the fact that the PNe images are from very different telescopes and researchers, it will be difficult to use any meaningful subsample. (4) In any case, to have a large enough number of circular PNe, all available circular PNe should be used.

In S97 I classified the PNe according to the type of binary interaction that may have shaped them: (i) Spherical $\mathrm{PNe}$, which I assumed in S97 to be PNe whose progenitors did not interact with any close companion, stellar or substellar. (ii) Bipolar PNe. Most, but not all, bipolar PNe belong to this class. In S97 I assumed that these are PNe whose progenitors interacted with close stellar companions outside their envelopes. (iii) Extreme-Elliptical PNe. This class includes some bipolar PNe, but mainly extreme-elliptical PNe, e.g., having a ring but not two lobes. I assumed in S97 that the progenitors of most of these PNe interacted with a stellar companion via a common envelope phase. (iv) Elliptical PNe. By elliptical alone, I refer to moderate elliptical PNe which show only small departure from sphericity. In S97 the progenitors of these PNe were assumed to have interacted with substellar objects, but not with a close stellar object. In a later paper (Soker 2001b) I argue that many PNe that belong to classes $(i i i)$ and $(i v)$ can be formed from the influence of a companion outside the progenitor's envelope, if the companion is not too close and/or not too massive, but still accretes and blows its own collimated fast wind (CFW).

In Table $1 \mathrm{I}$ compare the galactic distribution of the four classes listed by S97. The quantities that are given for each class are as follows. The total number of PNe in the class, $N_{\mathrm{T}}$, the average galactic longitude of all PNe in the class $l_{\mathrm{a}}$, and the number of $\mathrm{PNe}$ toward the galactic center, i.e., having $l<90^{\circ}$ or $l>270^{\circ}, N_{\text {in }}$. Also given are quantities just for PNe toward the galactic center: $l_{\text {in }}$ is the average value of the galactic longitude, $f b_{\text {in }}$ is the fraction of PNe having galactic latitude of $|b|>10^{\circ}$, and $\left.<b_{\text {in }}\right\rangle$ is the median value of $|b| . N_{\text {out }}$ is the number of PNe away from the galactic center, i.e., having $90^{\circ}<$ $l<270^{\circ}$. Other quantities with subscript "out" have the same meaning as those with subscript "in", but only for $\mathrm{PNe}$ away from the galactic center. For statistics involving the galactic longitude I took the galactic longitude of $\mathrm{PNe}$ with $180^{\circ}<l<360^{\circ}$ to be $l_{\mathrm{s}}=360^{\circ}-l$.

One well-known property and two new ones clearly emerge from the table.

(1) As is well known (see, e.g., reviews by Corradi 2000 and Manchado et al. 2000) bipolar PNe are concentrated toward the galactic plane, elliptical ones have intermediate scale height, while spherical PNe have the largest scale height. The relevant quantities in the table that show this behavior are $f b_{\text {in,out }}$ and $<b_{\text {in,out }}>$.

(2) The number of PNe towards and away from the galactic center in each class, $N_{\text {in }}$ and $N_{\text {out }}$, and the average galactic latitude $l_{\mathrm{a}}$, reveal that spherical $\mathrm{PNe}$ tend to be concentrated away from the galactic center. While the differences in the fraction of PNe away from the galactic center, $N_{\text {out }} / N_{\mathrm{T}}$, between the three classes of nonspherical $\mathrm{PNe}$ are within the statistical errors, the value for spherical PNe is more than twice as large. The selection effect due to galactic extinction is unknown, and may affect the results.

(3) Another new property is the relatively very high average galactic latitude of spherical PNe toward the galactic center. This is seen from comparing the values of $f b_{\text {in }}$ and $<b_{\text {in }}>$ with $f b_{\text {out }}$ and $<b_{\text {out }}>$. For the three classes of nonspherical PNe $f b_{\text {in }}<f b_{\text {out }}$ and $<b_{\text {in }}><<b_{\text {out }}>$, while for spherical PNe opposite inequalities hold. Another way to see this is by noticing that there is a smooth increase in $f b_{\text {out }}$ and $\left\langle b_{\text {out }}\right\rangle$ when moving from bipolar to extreme-elliptical to elliptical and to spherical, while in $f b_{\text {in }}$ and $<b_{\text {in }}>$ the smooth increase holds only for the nonspherical $\mathrm{PNe}$, with a sharp rise 
between elliptical and spherical PNe. Since spherical PNe in the sample used here tend to have low surface brightness, this specific jump may be due to extinction in the galactic plane. However, the general trend may be real. This behavior is less certain also because of the poor statistics, as there are only six spherical PNe toward the galactic center, and because the relevant physical quantity is the galactic height, for which the distance to each PN has to be known. I use the galactic latitude in the statistical analysis in order to avoid the large uncertainties in distances to PNe. Despite these drawbacks, in the next section I suggest that this behavior is real, and try to explain it.

As discussed in the previous section, Manchado et al. (1996, 2000) use a different classification for spherical PNe. In their classification properties (2) and (3) listed above are not found. As also mention in the previous section, I disagree with Manchado et al. (1996) in the classification of many PNe as spherical, since these contain elliptical substructures within the main circular shell. Further studies, with larger samples, are clearly needed here.

\subsection{Mass loss history}

From the structures of most $\mathrm{PNe}$, as well as other considerations, we know that most stars terminate the AGB by blowing a superwind (Renzini 1981), i.e., a final intensive wind (FIW), which is not faster than the regular AGB wind but has a much higher mass loss rate. In PNe this wind forms a dense shell. In many cases, inward to this shell there is a bright rim formed by the fast wind from the central star, and a fainter shell or halo outside the shell (Frank et al. 1990). Examining the images of the 18 spherical PNe (S97 Table 2), I find that most do not show signatures of a FIW (Soker 2000). I find dense shells only in Bd+30 3639 and IC 3568, both of which were suspected as being elliptical seen pole-on (marked PO by S97), H3-75, and possibly in A 15 and Lo 4. As discussed in Soker (2000), where more details and references can be found, the same correlation holds in many elliptical PNe; their outer faint halo is spherical, or only slightly elliptical, while the dense shell, which was formed from the FIW (superwind), is highly nonspherical. The positive correlation, albeit not perfect, between the onset of a FIW and a more nonspherical mass loss geometry is discussed by Soker (2000).

\section{What is required to become a spherical planetary nebula?}

\subsection{The metallicity connection}

I propose the following explanation for the findings of the previous section. To form a spherical PNe the progenitor should have a metallicity below a critical value. This is supported by the correlation between metallicity and morphology of PNe in the LMC, as presented in Figs. 1-3 of Stanghellini et al. (2000). For a given mass, the probability of forming a spherical PN increases as metallicity decreases, starting from zero probability at the critical metallicity $[\mathrm{Fe} / \mathrm{H}]_{\mathrm{c}}$. The star should also be above some lower mass limit. For a given metallicity, the probability of forming a spherical PN increases with the progenitor initial mass $M_{\mathrm{i}}$. I further argue, as I have been doing for the last decade, that this is expected if planets play a significant role in spinning-up evolved stars (Soker 2001a and references therein). To show that this is compatible with the galactic distribution of spherical PNe, I consider the galactic-metallicity dependence on age and the distance to the galactic center. Hereafter the age $t$ is expressed in Gyr (being positive with $t=0$ at present), while the distance to the galactic center, the Galactocentric radius, will be with respect to the distance of the sun to the galactic center in units of kpc $(r=0$ at the solar neighborhood, and $r>0$ moving away from the galactic center).

Lineweaver (2001) studies the relation between the metallicity of sun-like stars and the presence of hot Jupiter-like planets orbiting them. Lineweaver uses a galactic metallicity evolution in the solar neighborhood which presently has $[\mathrm{Fe} / \mathrm{H}]_{\mathrm{s}}=-0.2$, similar to the metallicity given by Allen et al. (1998) in their PNe study, but lower than values given by others, e.g., Carraro et al. (1998) and Rocha-Pinto et al. (2000; see reviews by Henry \& Worthey 1999 and Shields 2002). I take a metallicity evolution in the solar neighborhood to be some average of these studies,

$$
\begin{aligned}
{[\mathrm{Fe} / \mathrm{H}]_{\mathrm{S}}(t)=} & -0.1-0.02 t-a(t-5)^{4} \\
& \begin{cases}a=0 & t \leq 5 \\
a=5 \times 10^{-4} & 5<t \lesssim 10,\end{cases}
\end{aligned}
$$

where $t$ is the age given in Gyr, and subscript $s$ refers to the solar neighborhood. Allen et al. (1998) deduce from their PNe study that the metallicity gradient, with respect to the distance from the galactic center, was steeper in the past, while Carraro et al. (1998) find a nonmonotonic evolution of the metallicity gradient that, they argue, is compatible with a gradient that does not change with time. Again, I take the gradient evolution to be the average between these two studies,

$$
\frac{\mathrm{d}[\mathrm{Fe} / \mathrm{H}]}{\mathrm{d} r}=-2 \times 10^{-4} t^{2}-0.001 t-0.09 \mathrm{dex} \mathrm{kpc}^{-1}
$$

where $r$ is the distance from the galactic center minus the solar distance to the galactic center (in $\mathrm{kpc}$ ). The metallicity is therefore given by

$$
[\mathrm{Fe} / \mathrm{H}](t, r)=[\mathrm{Fe} / \mathrm{H}]_{\mathrm{s}}+\frac{\mathrm{d}[\mathrm{Fe} / \mathrm{H}]}{\mathrm{d} r} r
$$

The contour map of $[\mathrm{Fe} / \mathrm{H}](t, r)$ is plotted in Fig. 1 . The contour levels have a constant spacing of 0.1 dex, with values marked near each contour line.

For present purposes the exact values of the metallicity are not important, but only the general variation of metallicity with $r$ and $t$. To demonstrate the proposed explanation for the spherical $\mathrm{PNe}$ properties, I estimate the critical metallicity to be $[\mathrm{Fe} / \mathrm{H}]_{\mathrm{c}} \simeq-0.4$, and mark 
Table 1. Distribution of PNe in the galaxy.

\begin{tabular}{|c|c|c|c|c|}
\hline PNe Type & Spherical & Bipolar & E-Elliptical & Elliptical \\
\hline Source (S97) & Table 2 & Table 3 & Table 4 & Table 5 \\
\hline$N_{\mathrm{T}}$ & 18 & 49 & 111 & 274 \\
\hline$l_{\mathrm{a}}$ & $112^{\circ}$ & $70^{\circ}$ & $65^{\circ}$ & $68^{\circ}$ \\
\hline$N_{\text {in }}$ & 6 & 35 & 77 & 188 \\
\hline$l_{\text {in }}$ & $52^{\circ}$ & $44^{\circ}$ & $40^{\circ}$ & $39^{\circ}$ \\
\hline$f b_{\text {in }}$ & 0.67 & 0.09 & 0.19 & 0.27 \\
\hline$<b_{\text {in }}>$ & $\sim 12^{\circ}$ & $2^{\circ}$ & $4^{\circ}$ & $5^{\circ}$ \\
\hline$N_{\text {out }}$ & 12 & 14 & 34 & 86 \\
\hline$l_{\text {out }}$ & $142^{\circ}$ & $135^{\circ}$ & $124^{\circ}$ & $130^{\circ}$ \\
\hline$f b_{\text {out }}$ & 0.42 & 0.14 & 0.24 & 0.36 \\
\hline$<b_{\text {out }}>$ & $9^{\circ}$ & $4^{\circ}$ & $4.5^{\circ}$ & $7^{\circ}$ \\
\hline$N_{\text {out }} / N_{\mathrm{T}}($ all $)$ & $0.67(18)$ & $0.29(49)$ & $0.31(111)$ & $0.31(274)$ \\
\hline$N_{\text {out }} / N_{\mathrm{T}}\left(|b|>10^{\circ}\right)$ & $0.56(9)$ & $0.40(5)$ & $0.35(23)$ & $0.38(81)$ \\
\hline$N_{\text {out }} / N_{\mathrm{T}}\left(|b|>20^{\circ}\right)$ & $0.67(6)$ & $-(0)$ & $0.29(7)$ & $0.35(31)$ \\
\hline
\end{tabular}

The classification used is according to S97. E-Elliptical means extreme-elliptical PNe, which S97 assumed to be formed (in most cases) via interaction with a stellar companion that went through a common envelope phase. Bipolar were assumed by S97 to result from interaction with a close stellar companion outside the AGB envelope, while elliptical were assumed to result from interaction with substellar companions or wide stellar companions. The meaning of the symbols are: $N_{\mathrm{T}}$ and $l_{\mathrm{a}}$, total number and average galactic longitude of PNe in each class, respectively; $N_{\text {in }}$ and $l_{\text {in }}$, number of PNe toward the galactic center, i.e., having galactic longitude of $l<90^{\circ}$ or $l>270^{\circ}$, and their average value of the galactic longitude $l$; $f b_{\text {in }}$ and $<b_{\text {in }}>$, fraction of PNe having galactic latitude of $|b|>10^{\circ}$ and the median value of $|b|$ for PNe toward the galactic center. Quantities with subscript "out" have the same meaning but for PNe away from the galactic center, i.e., having $90^{\circ}<l<270^{\circ}$. $N_{\text {out }} / N_{\mathrm{T}}($ all $)$ is the ratio of the number of all PNe away from the galactic center to all PNe in each type of PNe, and for all PNe in the type; $N_{\text {out }} / N_{\mathrm{T}}\left(|b|>10^{\circ}\right)$ and $N_{\text {out }} / N_{\mathrm{T}}\left(|b|>20^{\circ}\right)$, are the same ratio but only for PNe with $|b|>10^{\circ}$ and $|b|>20^{\circ}$, respectively. The number in parenthesis follows that ratio is the number of PNe in the denominator of each ratio. For statistics involving the galactic longitude I took the galactic longitude of $\mathrm{PNe}$ with $180^{\circ}<l<360^{\circ}$ to be $l_{\mathrm{s}}=360^{\circ}-l$.

this contour by a thicker line. If only stars with $[\mathrm{Fe} / \mathrm{H}]<$ $[\mathrm{Fe} / \mathrm{H}]_{\mathrm{c}} \simeq-0.4$ can form spherical $\mathrm{PNe}$, it is clear from the figure that more spherical PNe will be located at larger distances from the galactic center, i.e., $r>0$, and they all will be descendants of low mass progenitors. These PNe occupy the upper left corner of Fig. 1, where the large age means that these PNe are formed from low mass stars. These low mass star progenitors imply that the galactic distribution of the descendant PNe will have a large average galactic latitude, as is indeed observed (Manchado et al. 2000). Moreover, the spherical PNe closer to the galactic center, $r<0$, with $[\mathrm{Fe} / \mathrm{H}] \gtrsim-0.4$, are on average lighter (older) than those farther away from the galactic center, $r>0$, meaning a larger average galactic latitude. This is compatible with the findings of Sect. 2 (compare $f b_{\text {in }}$ and $<b_{\text {in }}>$ with $f b_{\text {out }}$ and $<b_{\text {out }}>$, all given in Table 1).

To have $\sim 5-10 \%$ spherical $\mathrm{PNe}$ among all $\mathrm{PNe}$, the probability for forming a spherical PNe must increase substantially as metallicity decreases by $\sim 0.3-0.4$ dex from the critical value. In the case assumed here, it is from $[\mathrm{Fe} / \mathrm{H}]_{\mathrm{c}} \simeq-0.4$ to $[\mathrm{Fe} / \mathrm{H}] \simeq-0.8$. Two other relevant phenomena also change significantly when metallicity is changed by $\sim 0.3-0.4$ dex. The first is the presence of hot-Jupiter stars around sun-like stars. The several tens of extrasolar planets that have been found tend to orbit metal-rich stars. Lineweaver (2001) analyzes 32 hosts of Jupiter-like planets, and argues that the probability of a sun-like star hosting a hot Jupiter-like planet increases from $\lesssim 10 \%$ at $[\mathrm{Fe} / \mathrm{H}]=0.1$ to $\sim 90 \%$ at $[\mathrm{Fe} / \mathrm{H}]=0.4$, and to almost $100 \%$ at $[\mathrm{Fe} / \mathrm{H}]=0.6$ (see also Santos et al. 2001). This is further strengthen by new detection of planets. From 1200 stars discussed by Vogt et al. (2002; where more details can be found) 44 planets and 4 brown dwarfs have been detected, i.e., $3.7 \%$ of surveyed stars were found to have planets. For stars with metallicity above solar, $[\mathrm{Fe} / \mathrm{H}]>0$, the detection fraction is $\sim 2.6$ times that for the entire sample (Vogt et al. 2002). Reid (2002) argues that at $[\mathrm{Fe} / \mathrm{H}] \gtrsim 0.3$ most sun-like stars harbor planets around them.

The second phenomenon is the distribution of HB stars on the Hertzsprung-Russel diagram of globular clusters (the HB morphology). The distribution, e.g., the relative number of blue HB stars, varies from one globular 


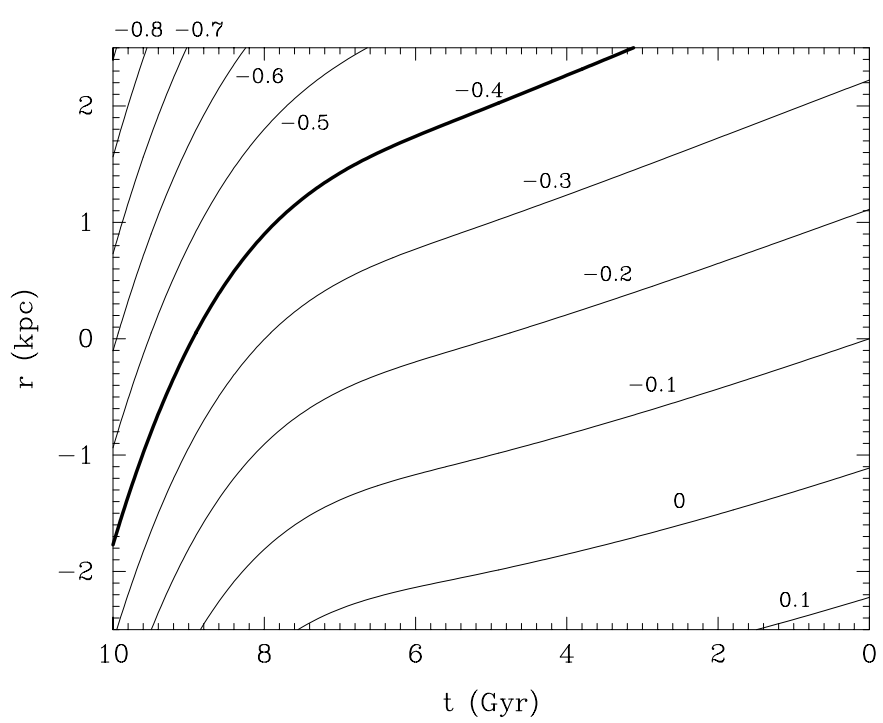

Fig. 1. Metallicity contour map. The value of $[\mathrm{Fe} / \mathrm{H}]$ is written near each contour line, $r$ is the Galactocentric distance relative to the sun ( $r=0$ for the solar neighborhood, $r>0$ is away from the galactic center) and $t$ is the age ( $t=0$ at present). This contour map is used in the text together with the distribution of spherical PNe in the galaxy (Table 1) to argue that the progenitors of spherical PNe have $[\mathrm{Fe} / \mathrm{H}] \lessgtr-0.4$ (marked by a thick line).

cluster to another. It has long been known that metallicity is the main, but not sole, factor which determines the HB morphology (for a historical review see, e.g., Rood et al. 1997; Fusi Pecci \& Bellazzini 1997). The other factor(s) which determine(s) the HB morphology is termed the "second parameter", and it is commonly thought that it has to do with mass loss on the red giant branch (Rood 1973). It is well known that the HB morphology significantly changes its behavior in a relatively narrow metallicity range of $-1.75 \lesssim[\mathrm{Fe} / \mathrm{H}] \lesssim-1.3$ (Soker \& Hadar 2002 and references therein). Therefore, it is not unlikely that the property which determines the probability of forming a spherical PN significantly changes over a narrow metallicity range, around $[\mathrm{Fe} / \mathrm{H}] \sim-0.5$.

\subsection{The planets conjecture}

I now relate the metallicity dependence on time and Galactocentric radius to the planet-induced axisymmetrical mass loss model. In this model low departure from spherical mass loss of some stars on the upper AGB is assumed to result from planets or brown dwarfs, which spin-up the AGB stellar envelopes (Soker 2001a and references therein). In other stars the nonspherical mass loss is due to interaction with stellar companions. Therefore, it is assumed in the model that if a massive enough and close enough planet is present around a star, the stellar envelope will be spun-up by the planet via tidal effects and common envelope evolution. The maximum orbital separation for an interaction to take place is $\sim 4 \mathrm{AU}$. In a previous paper (Soker 2001a) I argue that even planets having masses as small as $0.01 M_{\mathrm{J}}$, where $M_{\mathrm{J}}$ is Jupiter's mass, can lead to a slightly nonspherical mass loss geometry. Low mass stars hosting a close planet will engulf the planet already on the first giant branch (RGB), prior to the HB. The stars will be spun-up, their mass loss rate is likely to increase (Soker \& Hadar 2002), and they will retain very small mass in their envelopes, forming blue HB stars. Therefore, it is very likely that most known extrasolar systems will not form $\mathrm{PN}$ at all, but rather the hosting stars will engulf the orbiting planets during their RGB, lose most of their envelope on the RGB, and turn into blue HB stars. Blue HB stars are not likely to reach the upper AGB and form PNe because of their very low envelope masses. Even single low mass stars may lose too much mass on the RGB, possibly due to fast rotation, and never form PNe. For different reasons, Allen et al. (1998) argue that less than half of all stars of initial mass $M_{\mathrm{i}}<1.3 M_{\odot}$ form $\mathrm{PNe}$.

It seems therefore that stars hosting close planets with mass $\gtrsim 0.01 M_{\mathrm{J}}$ will either lose most of their envelope on the RGB and never form $\mathrm{PNe}$, or reach the AGB but form nonspherical, i.e., elliptical, PNe. To form a spherical PN a star should not have any close planet with a mass $\gtrsim 0.01 M_{\mathrm{J}}$, and of course no close stellar companion or brown dwarf either. It is very likely, e.g., Lineweaver (2001), Santos et al. (2001), and Reid (2002), that the probability of forming a planet, and the planet's mass, strongly depend on metallicity. (In a recent paper Murray \& Chaboyer 2002, on the other hand, argue that accretion of 6 Earth masses of iron rich material can explain most of the observations, with no need to assume a strong dependance of planets formation on metallicity.) Lineweaver (2001), for example, assumes that Earth-type planets are formed only when $[\mathrm{Fe} / \mathrm{H}] \gtrsim-1$, with increasing probability for increasing metallicity. For planets to induce mass loss in globular clusters, low mass planets should be formed already at $[\mathrm{Fe} / \mathrm{H}] \sim-1.7$. The initial mass of globular clusters stars now reaching the $\mathrm{HB}$ is $\sim 0.9$, so Earth-like planets maybe enough in some cases to enhance mass loss. More massive planets may be required to prevent stars of $M_{\mathrm{i}} \gtrsim 1.1 M_{\odot}$ from reaching the AGB. It is not unlikely that planets with mass of $\gtrsim 0.01-0.1 M_{\mathrm{J}}$ will be formed at high probability when $[\mathrm{Fe} / \mathrm{H}] \gtrsim-0.4$, as I suggested in the previous subsection. Such close planets will either cause their hosting star to form an elliptical PN or cause the star to lose most of its envelope, never forming a $\mathrm{PN}$.

\section{Summary}

The main finding of the paper concerns the distribution of spherical PNe in the galaxy. I found that spherical PNe tend to be concentrated away from the galactic center, and spherical PNe that are closer than the sun to the galactic center have a relatively very high average galactic latitude (Table 1). These trends should be confirmed, or rejected, when larger samples of well-resolved PNe become available, e.g., the Edinburgh/AAO/Strasbourg Catalogue of 
Galactic PNe (Parker et al. 2002; under construction). I used this finding to argue that spherical PNe are formed from low metallicity stellar progenitors (see Fig. 1). This correlation is observed directly in the LMC (Stanghellini et al. 2000). A crude estimate suggests that spherical $\mathrm{PNe}$ are formed from stars with $[\mathrm{Fe} / \mathrm{H}] \lesssim-0.4$ (and initial mass of $M_{\mathrm{i}} \gtrsim 0.9 M_{\odot}$ ), although only a minority of stars with these properties do form spherical PNe.

I further argued that the dependence on metallicity may result from the role played by planets in spinning-up the envelope of RGB and AGB stars. A planet will either spin-up the stellar envelope to blow a non spherical wind, hence forming an elliptical $\mathrm{PN}$, or, if the spin-up occurs on the RGB of a low mass star, the relatively fast rotating RGB star will lose most of its envelope and form a blue HB star, but will never reach the upper AGB and form a PN. Lineweaver (2001) finds the probability of forming Jupiter-like planets, with masses of $M_{\mathrm{p}} \sim M_{\mathrm{J}}$, to increase from $\lesssim 10 \%$ at $[\mathrm{Fe} / \mathrm{H}]_{1}=0.1$ to $\sim 90 \%$ at $[\mathrm{Fe} / \mathrm{H}]_{\mathrm{u}}=0.4$ (see also Reid 2002). The planet-induced axisymmetrical mass loss model to explain the properties of spherical $\mathrm{PNe}$ requires that the probability of forming planets of $M_{\mathrm{p}} \sim$ $0.01-0.1 M_{\mathrm{J}}$ increase significantly from $[\mathrm{Fe} / \mathrm{H}]_{1} \simeq-0.8$ to $[\mathrm{Fe} / \mathrm{H}]_{\mathrm{u}} \simeq-0.4$. Of course, more massive planets can be formed as well. For comparison, the planet second parameter model to explain some HB morphologies of globular clusters (Soker \& Hadar 2002) requires that the probability of forming planets of $M_{\mathrm{p}} \simeq 0.01 M_{\mathrm{J}}$ increases significantly from $[\mathrm{Fe} / \mathrm{H}]_{\mathrm{l}} \simeq-1.7$ to $[\mathrm{Fe} / \mathrm{H}]_{\mathrm{u}} \simeq-1.3$. These three critical metallicity bands show a monotonic trend, which, I claim, supports the conjecture that planets play a crucial role in the mass loss history of evolved stars.

Acknowledgements. I thank the anonymous referee for clarifying comments. This research was supported in part by a grant from the US-Israel Binational Science Foundation.

\section{References}

Allen, C., Carigi, L., \& Peimbert, M. 1998, ApJ, 494, 247

Carraro, G., Ng, Y. K., \& Portinari, L. 1998, MNRAS, 296, 1045
Corradi, R. L. M. 2000, in Asymmetrical Planetary Nebulae II: From Origins to Microstructures, ed. J. H. Kastner, N. Soker, \& S. Rappaport, ASP Conf. Ser., 199, 25

Frank, A., Balick, B., \& Riley, J. 1990, AJ, 100, 1903

Fusi Pecci, F., \& Bellazzini, M. 1997, in The Third Conference on Faint Blue Stars, ed. A. G. D. Philip, J. Liebert, R. Saffer, \& D. S. Hayes (L. Davis Press), 255 [astro-ph/9701026]

Henry, R. B. C., \& Worthey, G. 1999, PASP, 111, 919

Lineweaver, C. H. 2001, Icarus, 151, 307 [astro-ph/0012399]

Manchado, A., Guerrero, M., Stanghellini, L., \& Serra-Ricart, M. 1996, The IAC Morphological Catalog of Northern Galactic Planetary Nebulae (Tenerife: IAC)

Manchado, A., Villaver, E., Stanghellini, L., \& Guerrero, M. A. 2000, in Asymmetrical Planetary Nebulae II: From Origins to Microstructures, ed. J. H. Kastner, N. Soker, \& S. Rappaport, ASP Conf. Ser., 199, 17

Marcy, G. W., \& Butler, R. P. 2000, PASP, 112, 137

Murray, N., \& Chaboyer, B. 2002, preprint [astro-ph/0106294]

Parker, Q. A., et al. 2002, in Planetary Nebulae, IAU 209, in press

Reid, I. N. 2002, PASP, in press [astro-ph/0112402]

Renzini, A. 1981, in Effects of Mass Loss on Stellar Evolution, ed. C. Chiosi, \& R. Stalio (Dordrecht: Reidel), 319

Rocha-Pinto, H. J., Maciel, W. J., Scalo, J., \& Flynn, C. 2000, A\&A, 358, 850

Rood, R. T. 1973, ApJ, 184, 815

Rood, R. T., Whitney, J., \& D'Cruz, N. 1997, in Advances in Stellar Evolution, ed. R. T. Rood, \& A. Renzini (Cambridge: Cambridge U. Press), 74

Santos, N. C., Israelian, G., \& Mayor, M. 2001, A\&A, 373, 1019

Shields, G. A. 2002, Rev. Mex. A \& A, 12 (Serie de Conferencias), Ionized Gaseous Nebulae, ed. W. Henney et al., 189

Soker, N. 1997, ApJS, 112, 487 (S97)

Soker, N. 2000, in Asymmetrical Planetary Nebulae II: From Origins to Microstructures, ed. J. H. Kastner, N. Soker, \& S. Rappaport, ASP Conf. Ser., 199, 71 [astro-ph/9909258]

Soker, N. 2001a, MNRAS, 324, 699 [astro-ph/0006362]

Soker, N. 2001b, ApJ, 558, 157

Soker, N., \& Hadar, R. 2002, MNRAS, 331, 731

Stanghellini, L., Shaw, R. A., Balick, B., \& Blades, J. C. 2000, ApJ, 534, L167

Vogt, S. S., Butler, R. P., Marcy, G. W., et al. 2002, ApJ, 568, 352 